\title{
Economic evaluation for the UK of nab-paclitaxel plus gemcitabine in the treatment of metastatic pancreas cancer
}

\author{
M Gharaibeh ${ }^{1}$, A McBride ${ }^{2}$, J L Bootman ${ }^{1}$ and I Abraham*,1,2 \\ ${ }^{1}$ Center for Health Outcomes and PharmacoEconomic Research, College of Pharmacy, University of Arizona, Tucson, AZ 85721, \\ USA and ${ }^{2}$ Arizona Cancer Center, University of Arizona, Tucson, AZ 85721, USA
}

Background: The combination of nab-paclitaxel plus gemcitabine (NAB-P + GEM) has shown superior efficacy over GEM monotherapy in metastatic pancreas cancer (MPC). Independent cost-effectiveness/utility analyses of NAB-P + GEM from the payer perspective have not been conducted for the UK.

Methods: A Markov model simulating the health outcomes and total costs was developed to estimate the life years gained (LYG) and quality-adjusted life years gained (QALY) and incremental cost-effectiveness (ICER) and cost-utility ratios (ICUR) for patients with MPC in a base case and in a probabilistic (PSA) sensitivity analysis. Total cost included the cost of supportive care medications, administration, chemotherapy, disease monitoring, and adverse reactions; and was discounted at 3.5\% per year. A full lifetime horizon and third party payer perspective was chosen.

Results: The total cost of NAB-P + GEM was $£ 5466$ higher than the cost for GEM. Respectively, LYGs were 0.97 vs 0.79 and QALYs were 0.52 vs 0.45 , with ICER of $£ 30367 / L Y G$ and ICUR of $£ 78086 /$ QALY, confirmed by PSA.

Conclusions: The superior survival efficacy of NAB-P + GEM over GEM in the management of MPC is associated with positive cost-effectiveness and cost-utility.

Pancreatic cancer is the tenth most common cancer and the fifth most common cause of cancer death in the United Kingdom (UK). In 2011 in the UK, 8773 new cases were diagnosed and 8320 patients with pancreatic cancer died. Though based on United States (US) data, between 46\% (American College of Surgeons, 2013) and 53\% (Siegel et al, 2014) of incident cases presented with metastatic pancreatic cancer (MPC). In the UK, MPC has a median survival of about 12 weeks (Cancer Research UK, 2012), 1-year survival rate of $18.0 \%$, and 5 -year rate of $3.6 \%$.

Gemcitabine (GEM) has been the standard chemotherapy for MPC since 1997, after a randomised trial of GEM against 5fluorouracil produced significant improvements in disease-related symptoms and prolonged median survival from 4.4 to 5.7 months (Burris et al, 1997). Subsequent trials of biological or cytotoxic agents in combination with GEM compared with GEM alone in MPC have not shown improved survival outcomes, with the exception of taxanes. In a phase II trial of weekly $1000 \mathrm{mg} \mathrm{m}^{-2}$
GEM in combination with twice-weekly EndoTAG-1, a cationic lipid-complexed paclitaxel particle, at either 11, 22, or $44 \mathrm{mg} \mathrm{m}^{-2}$ against GEM alone was found to improve median survival by 4.1, 4.6, and 4.4 months, respectively, compared to 2.7 months for GEM alone (Löhr et al, 2012).

Albumin-based paclitaxel (nab-paclitaxel (NAB-P); Celgene, Summit, NJ, USA) is classified as an anti-microtubule agent. It stabilises microtubules by preventing depolymerisation and thus blocks the reorganisation process of the microtubule network, which is essential for cell division leading to cell apoptosis. It was developed to avoid toxicities that arise from the parent medication such as solvent-related hypersensitivity reactions (Electronic Medicines Compendium, 2014).

NAB-P has been shown to be superior to paclitaxel in cancer types that overexpress osteonectin (SPARC, BM-40) such as melanoma and breast and lung carcinoma. As osteonectin is overexpressed in pancreatic cancer specimens, NAB-P was hypothesised to be 
potentially effective in MPC. The combination of nab-paclitaxel and GEM (NAB-P + GEM) as first-line treatment in MPC was first studied in a phase I/II clinical trial to determine the maximum tolerated dose and to provide efficacy and safety data. The maximum tolerated dose was defined as $125 \mathrm{mg} \mathrm{m}^{-2}$ NAB-P in combination with $1000 \mathrm{mg} \mathrm{m}^{-2}$ of GEM on days 1,8 , and 15 , and repeated on a 28 -day cycle. Overall survival was 12.2 months and the 1-year survival of $48 \%$ was among the highest reported in a phase II trial in patients with MPC (Von Hoff et al, 2011)

A recent open-label, randomised, multi-centre, phase III trial assessed the efficacy of NAB-P + GEM $v s$ GEM alone in improving overall survival. Eight hundred sixty-one naïve patients with advanced metastatic pancreatic cancer were randomly assigned to receive either $125 \mathrm{mg} \mathrm{m}^{-2}$ of $N A B-P$ plus $1000 \mathrm{mg} \mathrm{m}^{-2}$ of GEM on days 1,8 , and 15 every 28 days or $1000 \mathrm{mg} \mathrm{m}^{-2}$ of GEM weekly for 7 weeks, and then on days 1, 8, and 15 every 28 days. Median treatment duration was 3.9 months for NAB-P + GEM vs 2.7 months for GEM alone. Median overall survival was 8.5 months in the NAB-P + GEM arm and 6.7 months in the GEM arm. Survival rates were 35 vs 22\%, respectively, at 12 months and 9 vs $4 \%$, respectively, at 24 months (both $P<0.05$ ). Median progression-free survival was 5.5 months in the NAB-P + GEM arm vs 3.7 months in the GEM arm, and the corresponding hazard ratio was 0.69 $(P<0.05)$. [Von Hoff et al, 2013]

The economic evaluation reported here aimed to estimate the cost-utility and cost-effectiveness NAB-P + GEM compared with GEM alone in the treatment of MPC from the perspective of a UK third party payer.

\section{MATERIALS AND METHODS}

Patients population. The modelled cohort utilised the sample characteristics from the phase III clinical trial of NAB-P + GEM (Von Hoff et al, 2013): adults (median age 63 years), recently diagnosed and histologically or cytologically confirmed metastatic adenocarcinoma of the pancreas, no prior chemotherapy for metastatic disease, and Karnofsky performance status score $\geq 70$. Eighty-four percent of patients had liver metastases, and $46 \%$ had at least three metastatic sites. Kaplan-Meier survival curves for the two treatment arms were available for 24 months for progressionfree survival (PFS) and 39 months for overall survival (OS). Minimum dose intensity was $75 \%$ for the NAB-P + GEM arm and $85 \%$ for the GEM arm.

Model structure. A literature-based state-transition Markov model was developed in which patients in each treatment arm are described by a set of disease status reflecting the treatment pathway and outcomes (see Figure 1 for the structure of the survival model). Patients start in the model at the initiation of chemotherapy. After the first cycle of chemotherapy, there are three possible next states, each with associated probabilities: staying in the same MPC disease stage until the next cycle (PFS); progressing to the next MPC disease stage; or death. Patients in the progressive disease state have two possible next states: further disease progression or death. This is repeated across all cycles until the end of the chemotherapy regimen or death.

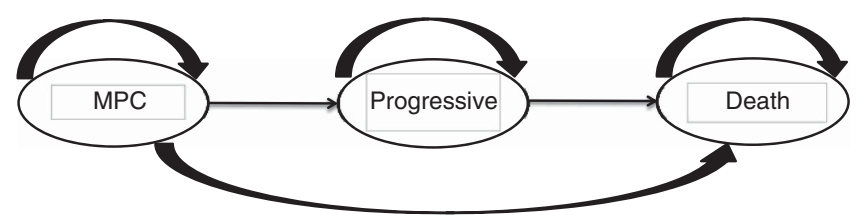

Figure 1. Structure of the survival model for advanced MPC.
The model was used to perform both cost-effectiveness analyses (cost per life year gained (LYG)) and cost utility analyses (cost per quality-adjusted life year (QALY)). The incremental cost-effectiveness ratio (ICER) and incremental cost utility ratio (ICUR) were calculated where ICER is the cost to achieve a one unit increase in life year and ICUR is the cost to achieve a one unit increase in QALY between alternatives.

The Markov model was specified in Microsoft Excel (Microsoft, Redmond, WA, USA) and included where necessary the use of Visual Basic Codes for probabilistic sensitivity analysis (PSA) and Cost Effectiveness Acceptability Curves. Data for the NAB-P + GEM and GEM arms were extracted from the Kaplan-Meier curves of the phase III trial using a digitising computer program

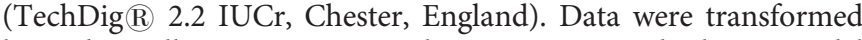
logarithmically to meet statistical assumptions, and a linear model was fitted using Microsoft Excel 2007 to estimate the parameters.

Cost and utility estimates used in model. Chemotherapy medications are dosed according to body surface area (BSA). A study on the average BSA of adult cancer patients in the UK estimated a BSA value of $1.83 \mathrm{~m}^{2}$ for patients with upper gastrointestinal/pancreas cancer (Sacco et al, 2010).

All costs were expressed in 2013 pound sterling (£). Drug acquisition costs were based on the British National Formulary (BNF) and cost per mg of chemotherapy, as necessary adjusted for wastage (The British National Formulary, 2014). The vial cost for $100 \mathrm{mg}$ of NAB-P was $£ 246.00$ and for $1000 \mathrm{mg}$ of GEM was $£ 162.76$. Medication cost was adjusted by using the relative dose intensities reported in the phase III clinical trial.

NHS Reference Costs (2012-2013) were used to estimate the cost of chemotherapy administration and the treatment of adverse drug reactions (Department of Health, 2013). Patients were assumed managed as day cases and not admitted to the hospital for drug administration. The cost of disease monitoring was based on the applicable health state and obtained per expert opinion. For a PFS health state on chemotherapy, disease monitoring was assumed to include one medical oncologist visit, one complete blood count and full biochemistry tests including electrolytes every 4 weeks, and one CA19-9 test every 8 weeks. Monitoring for a PFS health state off chemotherapy included the same lab tests but did not include a medical oncologist visit. For a disease progression health state, monitoring included for each applicable cycle, one medical oncologist visit, one complete blood count test, full biochemistry including electrolytes, one computerised tomography scan of more than three areas; one magnetic resonance imaging scan for more than three areas and CA19-9 test every month.

Vomiting, nausea, diarrhoea, stomatitis, rash, fatigue, anaemia, and peripheral neuropathy with grades III or IV were considered unlikely to require a full day of hospitalisation. They were treated as a day case admission. Febrile neutropenia was treated as requiring hospitalisation. The phase III clinical trial (Von Hoff et al, 2013) did not report cardiac side effects and hence these were not included in the model.

Health-related quality of life estimates based on the EuroQol (EQ-5D) were obtained online (Tam et al, 2013). The impact utility was assumed to last for one cycle. The health-related quality of life estimate was adjusted according to the number of cycles per year for each treatment: 12 cycles per year for the GEM alone arm and 13 cycles per year for the combination of NAB-P + GEM arm.

Costs and utilities incurred in the first year were not discounted. Those incurred after the first year were discounted at a 3.5\% rate.

Sensitivity analyses. One-way sensitivity analysis was performed using the upper and the lower confidence interval values for the selected parameters except for the nab-paclitaxel and gemcitabine vial costs where a plus or minus $50 \%$ value were used.

Probabilistic sensitivity analyses (PSA) were conducted on the model to take into account the simultaneous effect of uncertainty 
in model parameter values. This was achieved through repeated sampling of mean parameter values from a series of assigned distribution types, based on the point estimates and the standard error statistics for each average parameter value. Each set of samples from all the parameters generated a single estimate of expected costs, effects and net benefits generated by the model. Cost Effectiveness Acceptability Curves were plotted to show the probability that each treatment is cost-effective at various willingness-to-pay thresholds.

The PSA included covariance matrices that allowed joint distributions to be generated for PFS and OS. For both PFS and OS, Weibull two-parameter distributions using shape and scale were used as they fit the Kaplan-Meier empirical curves well. Constraints were added to ensure PFS was always shorter than OS (Table 1).

A normal distribution was applied to patients' BSA to reflect the variability that affects the calculation of chemotherapy drug exposure. The ranges of BSA sampled by the distribution reflect the likely variability in a population of patients with pancreatic cancer. Such uncertainty in the model leads to a variation in the cost of chemotherapy regimens in both treatment arms.

Uncertainty regarding the probability of AEs for those patients treated in both arms and health-related qualities of life were assessed using beta distributions. A constraint was added to assure that the utility for the progression-free health state is always better than the utility for the progressed health state.

\section{RESULTS}

The expected costs and outcomes of NAB-P + GEM and GEM in the UK are presented in Table 2. In the base case analysis, NAB$\mathrm{P}+\mathrm{GEM}$ treatment was more expensive than GEM treatment (£9314vs £3848) but yielded incremental benefits in terms of LYG (0.97 vs 0.79) and QALYs (0.52 vs 0.45). The ICER was $£ 30367 /$ LYG while the ICUR was $£ 78086 /$ QALY. The PSA confirmed the base case results: NAB-P + GEM was more expensive (£9320 vs $£ 3839)$ but yielded incremental benefits in terms of LYG (0.99 vs 0.80 ) and QALYs (0.54 vs 0.47 ) of the same order of magnitude. The PSA-generated ICER and ICUR were £28 847/LYG and $£ 78300$ /QALY, respectively. All of the 2000 simulations in the PSA confirmed the additional costs and incremental benefits for NAB-P + GEM over GEM (Figure 2) indicating that there is no probability of NAB-P + GEM having a poorer outcome than GEM. The Cost Effectiveness Acceptability Curves assess the uncertainty surrounding the mean ICER. The probability that NAB-P + GEM would be considered cost-effective compared with GEM alone was $50 \%$ for a threshold value of $£ 78000 /$ QALY and $70 \%$ for a threshold value of $£ 97000 /$ QALY (Figure 3).

One-way sensitivity analyses examined the impact of variability in vial costs and dose intensity on the results. Nab-paclitaxel vial cost was varied with a $50 \%$ increase or decrease and dose intensity with a $10 \%$ increase or decrease. Figure 4 shows the tornado diagram. The most influential parameter was NAB-P vial cost. A decrease in vial cost by $50 \%$ was associated with an ICUR of $£ 46402 / \mathrm{QALY}$.

Four gemcitabine vial sizes were used to evaluate the impact of wastage on our results: $200 \mathrm{mg}, 1,1.5$, and $2 \mathrm{~g}$ (Table 3). A vial calculation was made according to the upper and lower limits of

Table 1. NAB-P + GEM Kaplan-Maier curves parameters

\begin{tabular}{|l|c|c|c|}
\hline & Shape & Scale & Coefficient \\
\hline Progression-free survival \\
\hline Shape & 0.006703 & 0.000617231 & -4.68836 \\
Scale & 0.001955 & .370635 \\
\hline Overall survival & 0.002301 & & \\
\hline Shape & 0.000588 & 0.000160009 & -4.87509 \\
Scale &
\end{tabular}

gemcitabine dose, and the best combination was used. Nab-paclitaxel has only one vial size, which is $100 \mathrm{mg}$ per vial. Including the costs associated with product wastage had a relatively limited impact.

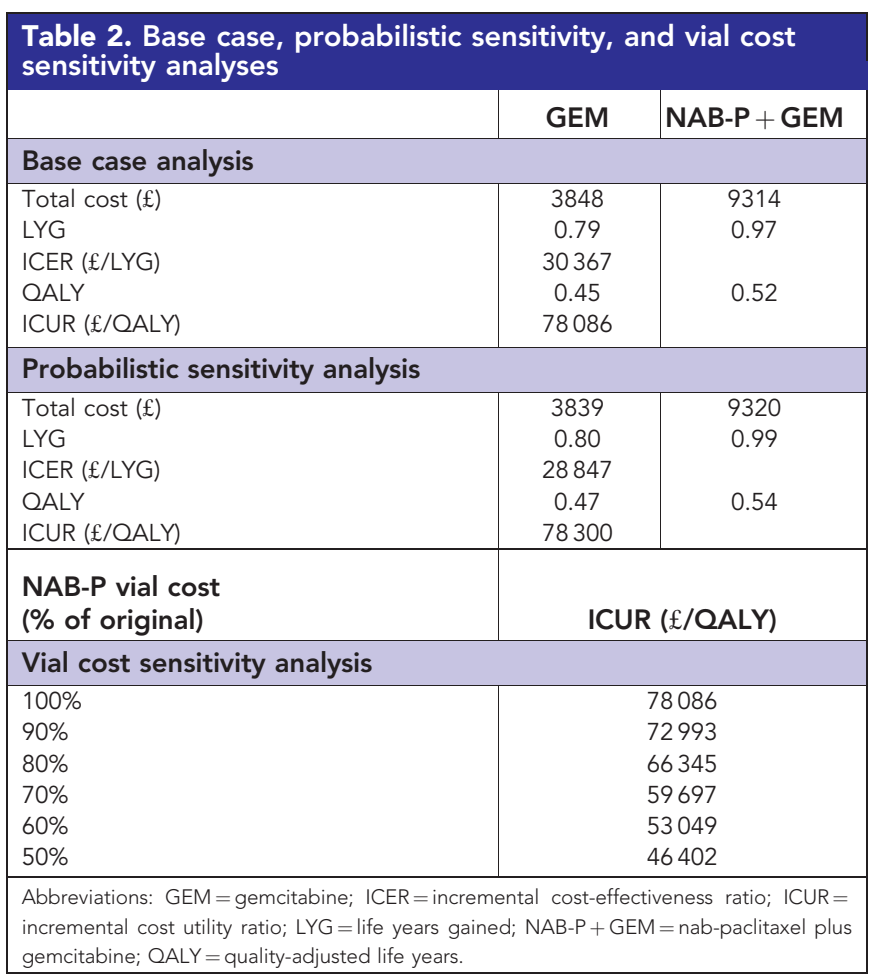

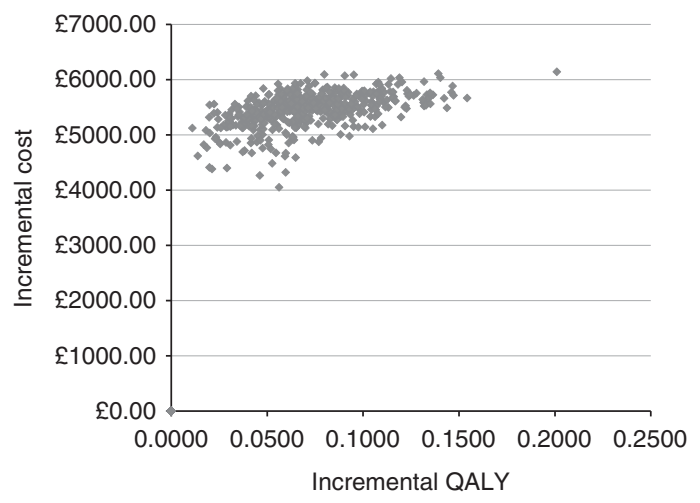

Figure 2. Scatter plot of probabilistic sensitivity analysis.

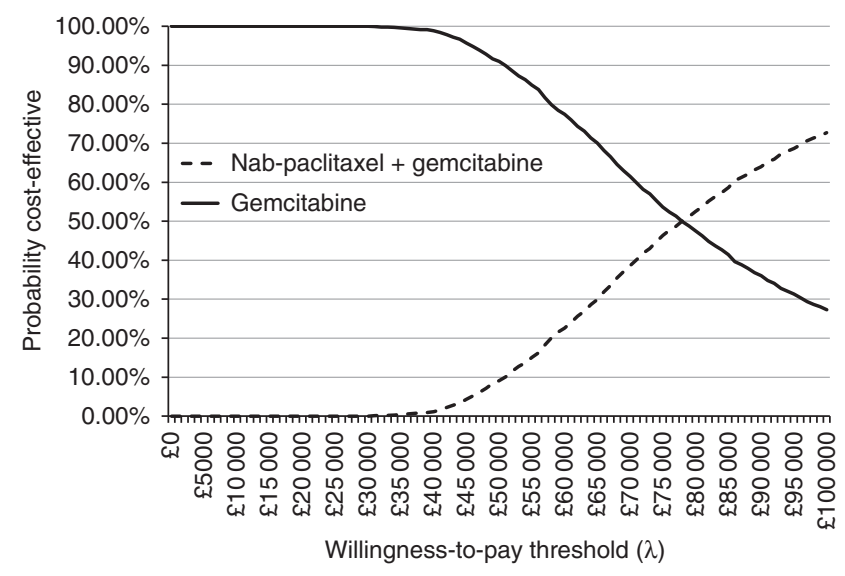

Figure 3. Cost-effectiveness acceptability curves. 


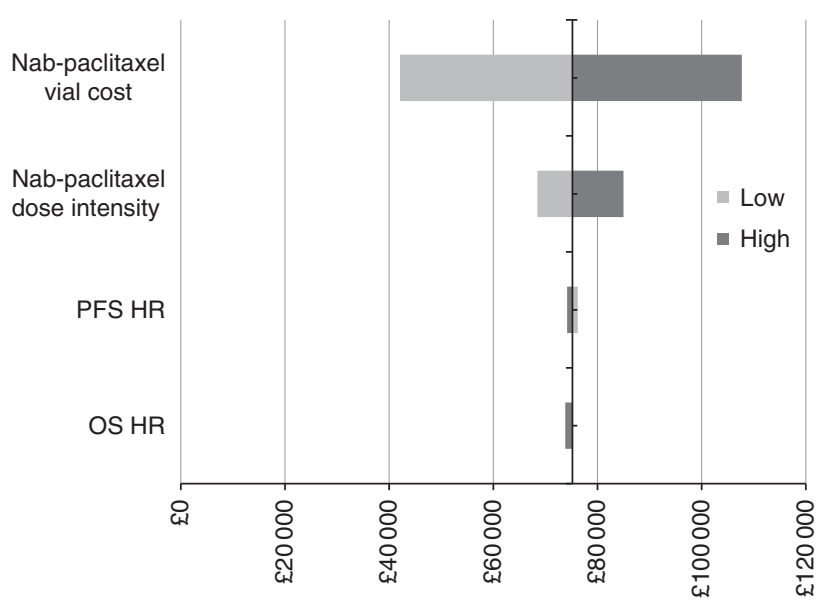

Figure 4. Tornado diagram.
Table 3. Cost of wastage and impact on outcome

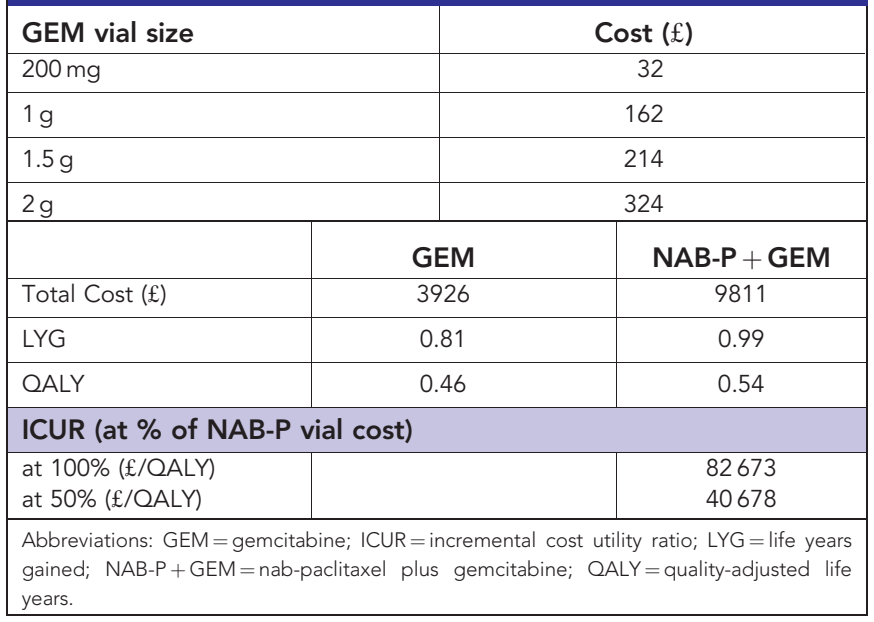

setting under consideration-metastatic pancreatic cancer-and the limited residual life expectancy of these patients. It is common practice in health technology assessment to value a treatment and decide about its reimbursement on the basis of a threshold value (e.g., $£ 30000 / \mathrm{QALY}$ in the UK and $\$ 50000 / \mathrm{Q} A L Y$ in the US), in which case NAB-P + GEM treatment would not be considered cost-effective and not be reimbursed. MPC is a case where absolute thresholds such as $£ 30000 / \mathrm{QALY}$ may not be clinically appropriate because of these patients' limited life expectancy compared with cases of earlier stage cancer, longer PFS, and/or longer OS.

In addition, there are more general arguments that caution against exceeding reliance on absolute thresholds to interpret the value of a treatment. As noted in a recently completed study supported by the European Union, inherent to the QALY is the assumption that modal patient preferences are consistent across health states and time when this may not be the case (European Consortium in Healthcare Outcomes and Cost-Benefit Research, 2013; Holmes, 2013). Neumann et al (2014) contend that the $\$ 50000 /$ QALY gained ratio may be misunderstood or misused, especially when it is applied to high-priced cancer and hepatitis $\mathrm{C}$ drugs. They also argue against determining a single threshold value representing a society's willingness-to-pay for QALYs gained, as diverse approaches may yield different ratios because of varying assumptions, contexts, or inferences-as our case of MPC underscores.

Further, the empirical base for the US \$50 000/QALY threshold and the corresponding $£ 30000 / \mathrm{QALY}$ remains elusive (Eichler et al, 2004). In a comprehensive review, Grosse puts the initial mention of the $\$ 50000$ threshold in 1972 at the initiation of Medicare coverage for patients with end-stage renal disease; the first article using a cost-effectiveness threshold in 1992 followed by the publication of the first studies using this threshold in 1995; and (informal) adoption of the threshold between 1996 and 1998 (Grosse, 2008). Using 1992 as reference and adjusting the then threshold of $\$ 50000 / \mathrm{QALY}$ with the US medical consumer price index, the threshold in 2012 would have been \$109000/QALY (Abraham et al, 2013) or approximately $£ 65000 / \mathrm{QALY}$ (at 50:30 conversion). A 2009 study placed the value of a quality human life at $\$ 129000$ ( $£ 77000$ ) using dialysis patients as the reference population (Lee et al, 2009). Again, this is under the assumption of consistency of (patient) preferences and (payer) willingness-to-pay across health states and time, without adjustment for metastatic disease or poor survival prognosis.

Although we focused our evaluation on cost-effectiveness and cost-utility, it also enables an initial assessment of the gross budget impact of treatment of MPC with NAB-P + GEM over GEM. In the base case analysis, the cost for treating one patient with NAB-P + GEM was estimated at $£ 9314$ and the cost differential 
over GEM at $£ 5466$. If $46 \%$ (American College of Surgeons, 2013) of the 8773 newly diagnosed pancreatic cancer patients in the UK in 2011, the proportion conservatively estimated to have metastatic disease, would have been a candidate for treatment with NAB-P + GEM, the gross budget impact would be $£ 37587392$ and the incremental budget impact over GEM treatment would be $£ 22058480$.

In general, higher rates of adverse events were observed in the $\mathrm{NAB}-\mathrm{P}+\mathrm{G}$ arm compared with the GEM arm, with proportionately more patients in the NAB-P + GEM treatment arm experiencing neutropenia, leukopenia, fatigue, and peripheral neuropathy (Von Hoff et al, 2013). This may have resulted in additional costs beyond direct medical treatment that we were not able to capture in our analysis. Other limitations of our study are that, in the absence of patient-level data, we were obliged to use the EQ-5D utility values obtained in a Canadian study that surveyed medical oncologists; that the payer perspective taken in our analysis does not address the societal benefit that may be accrued; and that we did not include other treatments such as FOLFIRINOX, which also has been shown to be have greater efficacy than GEM alone (Conroy et al, 2011).

Although we do not advocate a threshold-based approach to the economic valuation of NAB-P + GEM treatment, we do recognise the health technology assessment model prevailing in the UK. The National Institute for Health and Care Excellence, as well as similar organisations in other jurisdictions, may wish to customise its assessments when appraising treatments that may extend the life of patients with an incurable disease and a short life expectancy. This could be done by waiving thresholds or adopting higher thresholds. A perhaps more patient-centric approach would be to give a higher weight to QALYs achieved in the later stages of terminal disease, using the assumption that during the extended survival period, patients could experience the quality of life anticipated for a healthy individual of the same age.

Variations in the vial cost of NAB-P impacts the QALY estimates. Although, of course, lowering the price of any medication or treatment increases affordability and patient access, many variables determine the cost and price of a given treatment. One approach to lowering drug costs at the population level is by negotiating volume-based discounts.

\section{CONCLUSION}

The superior survival efficacy of NAB-P + GEM over GEM in the management of MPC is associated with positive cost-effectiveness and cost-utility.

\section{REFERENCES}

Abraham I, Harrington AR, Bootman JL (2013) The QALY controversy in the United States. Invited presentation at the EU Special Conference "Health Outcomes in Europe: the QALY controversy. New European Guidelines in Cost-Effectiveness Assessment". Available from: http://www. echoutcome.eu/images/stories/Abraham_QALY_Controversy_in_US.pdf (Accessed 28 December 2014).

American College of Surgeons (2013) National cancer data base public benchmarks. Available from: https://cromwell.facs.org/BMarks/BMPub/ ver10/Docs (Accessed 28 December 2014).

Burris HA, Moore MJ, Andersen J, Green MR, Rothenberg M, Modiano MR, Cripps MC, Portenoy RK, Storniolo AM, Tarassoff P, Nelson R, Dorr FA, Stephens CD, Von Hoff DD (1997) Improvements in survival and clinical benefit with gemcitabine as first-line therapy for patients with advanced pancreas cancer: a randomized trial. J Clin Oncol 15: 2403-2413.

Cancer Research UK (2012) Pancreatic cancer survival statistics. Available from: http://www.cancerresearchuk.org/cancer-info/cancerstats/types/ pancreas/survival/ (Accessed 28 December 2014).
Conroy T, Desseigne F, Ychou M, Bouche O, Guimbaud R, Becouarn Y, Adenis A, Raoul JL, Gourgou-Bourgade S, de la Fouchardiere C, Bennouna J, Bachet JB, Khemissa-Akouz F, Pere-Verge D, Delbaldo C, Assenat E, Chauffert B, Michel P, Montoto-Grillot C, Ducreux M (2011) FOLFIRINOX $v s$ gemcitabine for metastatic pancreatic cancer. $N$ Engl $J$ Med 364: 1817-1825.

Department of Health (2013) NHS reference costs 2012 to 2013. Available from: https://www.gov.uk/government/publications/nhs-reference-costs2012-to-2013 (Accessed 28 December 2014).

Eichler HG, Kong SX, Gerth WC, Mavros P, Jonsson B (2004) Use of costeffectiveness analysis in health-care resource allocation decision-making: how are cost-effectiveness thresholds expected to emerge? Value Health 7: $518-528$.

Electronic Medicines Compendium (2014) Summary of product characteristics (SPC) Abraxane $5 \mathrm{mg} / \mathrm{ml}$ powder for suspension for infusion. Available from: http://www.medicines.org.uk/EMC/medicine/21384/SPC/Abraxane + $5+\mathrm{mg}+\mathrm{ml}+$ powder + for + suspension + for + infusion/\%20 Accessed (Last accessed 28 December 2014).

European Consortium in Healthcare Outcomes and Cost-Benefit Research (2013) Final report summary. Available from: http://cordis.europa.eu/ result/rcn/57938_en.html (Accessed 28 December 2014).

Grosse SD (2008) Assessing cost-effectiveness in healthcare: history of the \$50,000 per QALY threshold. Exp Rev Pharmacoecon Outcomes Res 8: $165-178$.

Holmes D (2013) Report triggers quibbles over QALYs, a staple of health metrics. Nat Med 19: 248.

Lee CP, Chertow GM, Zenios SA (2009) An empiric estimate for the value of life: updating the renal dialysis cost-effectiveness standard. Value Health 12: $80-87$.

Löhr J, Haas S, Bechstein W, Bodoky G, Cwiertka K, Fischbach W, Folsch U, Jager D, Osinsky D, Prausova J, Schmidt W, Lutz M (2012) Cationic liposomal paclitaxel plus gemcitabine or gemcitabine alone in patients with advanced pancreatic cancer: a randomized control phase II trial. Ann Oncol 23: 1214-1222.

National Institute for Health and Care Excellence (NICE) (2010) Final Appraisal Determination. Paclitaxel as albumin-bound nanoparticles in combination with gemcitabine for previously untreated metastatic pancreatic cancer. http://www.nice.org.uk/guidance/gid-tag453/ documents/pancreatic-adenocarcinoma-untreated-metastatic-paclitaxelalbuminbound-nanoparticles-with-gemcitabine-id680-final-appraisaldetermination-document2 (Accessed 7 February 2015).

Neumann P, Joshua T, Weinstein M (2014) Updating cost-effectiveness - the curious resilience of the \$50,000-per-QALY threshold. N Engl J Med 9: 796-797.

Sacco JJ, Botten J, Macbeth F, Bagust A, Clark P (2010) The average body surface area of adult cancer patients in the UK: A Multicentre Retrospective Study. PLoS One 5: e8933.

Siegel R, Ma J, Zou Z, Jemal A (2014) Cancer statistics, 2014. CA Cancer J Clin 64: 9-29.

Tam V, Ko Y, Mittmann N, Cheung M, Kumar K, Hassan S, Chan K (2013) Cost-effectiveness of systemic therapies for metastatic pancreatic cancer. Curr Oncol 20: e90-e106.

The British National Formulary (2014) Available from: https:// cromwell.facs.org/BMarks/BMPub/ver10/Docs http://bnf.org/bnf/ (Accessed 25 February 2014).

Von Hoff DD, Ervin T, Arena FP, Chiorean EG, Infante J, Moore M, Seay T, Tjulandin SA, Ma WW, Saleh MN, Harris M, Reni M, Dowden S, Laheru D, Bahary N, Ramanathan RK, Tabernero J, Hidalgo M, Goldstein D, Van Cutsem E, Wei X, Iglesias J, Renschler MF (2013) Increased survival in pancreatic cancer with nab-paclitaxel plus gemcitabine. N Engl J Med 369: 1691-1703.

Von Hoff DD, Ramanathan RK, Borad MJ, Laheru DA, Smith LS, Wood TE, Korn RL, Desai N, Trieu V, Iglesias JL, Zhang H, Soon-Shiong P, Shi T, Rajeshkumar NV, Maitra A, Hidalgo M (2011) Gemcitabine plus nab-paclitaxel is an active regimen in patients with advanced pancreatic cancer: a phase I/II trial. J Clin Oncol 29: 4548-4554.

This work is published under the standard license to publish agreement. After 12 months the work will become freely available and the license terms will switch to a Creative Commons AttributionNonCommercial-Share Alike 4.0 Unported License. 